\title{
Estereotipos Sobre los Mapuches: Su Reciente Evolución
}

\section{Stereotypes About Mapuches: Recent Evolution}

\author{
José L. Saiz y M. Eugenia Rapimán \\ Universidad de La Frontera
}

\author{
Antonio Mladinic \\ Pontificia Universidad Católica de Chile
}

\begin{abstract}
Mediante una réplica y ampliación de un estudio realizado en 1988 por Saiz (1991), se examinó la evolución de los estereotipos que estudiantes universitarios no indígenas atribuyen a dos categorías cronológicas de mapuches: pretéritos y contemporáneos. Este examen fue realizado según el contenido, favorabilidad, notoriedad y uniformidad de los estereotipos. Mediante un cuestionario, 407 participantes asignaron atributos a las dos categorías de mapuches. Confirmando lo esperado, los estereotipos replican, en general, aquellos reportados por Saiz (1991) y surgen, además, dos nuevos estereotipos desfavorables sugeridos en la literatura. La preservación de los estereotipos es discutida mediante el enfoque de subtipos y subgrupos (Richards \& Hewstone, 2001), en tanto que el surgimiento de nuevos estereotipos es analizado desde la teoría del conflicto realista (Campbell, 1965).
\end{abstract}

Palabras clave: estereotipos, indígenas, evolución.

Replicating and expanding a study by Saiz (1991), we examined the evolution of the stereotypes that non-indigenous university students ascribe to two distinctive Mapuche chronological categories: past and present. The content, favorability, salience, and uniformity of the stereotypes were analyzed. Using a questionnaire, 407 participants assigned attributes to both Mapuche categories. As expected, almost all the stereotypes replicated those reported by Saiz (1991) and, at the same time, two new unfavorable stereotypes emerged. The persistence of stereotypes is discussed according to the process of subtyping and subgruping (Richards \& Hewstone, 2001). The development of new stereotypes is analyzed from the realistic conflict theory perspective (Campbell, 1965).

Keywords: stereotypes, natives, evolution.

Antecedentes

Enfatizando los aspectos esenciales del concepto (para una revisión reciente ver Schneider, 2005), un estereotipo puede ser definido como un conjunto de creencias que los miembros de un grupo (endogrupo) comparten acerca de los atributos (e.g., trabajador o desconfiado) que caracterizan a los miembros de otro grupo (exogrupo). En el presente estudio

\footnotetext{
José L. Saiz, Departamento de Psicología, Universidad de La Frontera, Temuco, Chile.

M. Eugenia Rapimán, Departamento de Psicología, Universidad de La Frontera, Temuco, Chile.

Antonio Mladinic, Escuela de Psicología, Pontificia Universidad Católica de Chile, Santiago, Chile.

La correspondencia relativa a este artículo debe ser dirigida a José L. Saiz, Departamento de Psicología, Universidad de La Frontera, Casilla 54-D, Temuco, Chile. E-mail: jsaiz@ufro. $\mathrm{cl}$

Esta investigación fue financiada por el Fondo Nacional de Desarrollo Científico y Tecnológico (FONDECYT), a través del Proyecto No 1060395.
}

se examinaron los estereotipos que estudiantes no indígenas de la Universidad de La Frontera (UFRO) asignan a los indígenas mapuches. Específicamente, se realizó una réplica y ampliación de una investigación sobre estereotipos mapuches realizada por Saiz $(1991)^{1}$ en 1988, en esa misma población estudiantil. En la réplica se indagó la continuidad y cambio de los estereotipos a través del tiempo, en tanto que en la ampliación se pesquisó el surgimiento de nuevos estereotipos.

Saiz (1991) empleó varias dimensiones para describir los estereotipos obtenidos, entre otras, contenido, favorabilidad, notoriedad y uniformidad. El contenido es definido por los atributos específicos que son asignados a un grupo (Fiske, Cuddy, Glick $\& \mathrm{Xu}, 2002)$. Estos atributos suelen organizarse en estructuras relativamente diferenciadas y complejas que corresponden a los distintos estereotipos ads-

\footnotetext{
1 En Saiz y Williams (1991) y Saiz (2002) se encuentran ver-
} siones resumidas y en castellano de ese estudio. 
critos a ese grupo. A su vez, estas configuraciones de atributos pueden organizarse en estructuras de orden superior que, como se verá, reforzarían la continuidad temporal de los estereotipos.

La favorabilidad de un estereotipo puede ser entendida como el grado de deseabilidad social de sus atributos (Nelson, 2006). Así, pueden haber estereotipos más favorables o desfavorables en función, respectivamente, de una mayor o menor deseabilidad social de los atributos. La notoriedad, también llamada generalización (Schneider, 2005), es definida como el grado en que el endogrupo percibe que un estereotipo caracteriza a los miembros del exogrupo. Un estereotipo es notorio cuando sus atributos son aplicables a porciones sustanciales del exogrupo. Finalmente, la uniformidad del estereotipo se refiere al nivel de acuerdo entre los miembros de un grupo de que el estereotipo se aplica a otro grupo (Devine \& Elliot, 1995). La uniformidad refleja la homogeneidad de las creencias estereotípicas dentro del endogrupo.

\section{El Estudio a Ser Replicado y Ampliado}

Mediante un análisis factorial de datos obtenidos en una muestra de estudiantes no indígenas de pregrado de la UFRO $(n=200)$, Saiz (1991) encontró nueve estereotipos (factores) diferenciados según dos categorías cronológicas de mapuches: pretéritos y contemporáneos.

\section{Estereotipos sobre los Mapuches Pretéritos}

Cinco estereotipos aparecieron ligados a estos mapuches. El primero, rotulado valientes guerreros, incluyó los atributos fuertes, rápidos, valientes, inteligentes, leales y amaban la libertad. Mediante este estereotipo favorable se asigna a los mapuches una disposición a realizar hazañas marcadas por el valor, la astucia y el vigor, reflejando una visión romántica de ellos como héroes militares de antaño. Un segundo estereotipo, denominado indios hostiles, adscribe a los mapuches una tendencia a oponerse a otros tanto en actitudes como en acciones. Este estereotipo desfavorable incluyó los atributos violentos, porfiados y desconfiados. Un tercer estereotipo contuvo los atributos honrados, responsables, leales e inteligentes, todos positivos. Este estereotipo fue llamado gente noble y refleja una imagen favorable de los mapuches como personas dignas y respeta- bles. Un cuarto estereotipo, de contenidos favorables, fue denominado indios industriosos, ya que implica percibir a los mapuches como trabajadores, responsables y expresivos. Finalmente, el quinto estereotipo ligado a los mapuches pretéritos incluyó los atributos desfavorables no querían educarse y no querían ser chilenos. El rótulo indios que resistían la chilenización fue empleado para denominar este estereotipo, el cual asigna a los mapuches una disposición a rechazar los intentos de asimilación de la sociedad mayoritaria, esto es, no indígena.

\section{Estereotipos sobre los Mapuches Contemporáneos}

Cuatro estereotipos surgieron asociados a estos mapuches. El primero, llamado buenos ciudadanos, refleja una imagen positiva de ciudadanos motivados, capaces y probos. Este estereotipo incluyó 12 atributos, a saber: responsables, inteligentes, trabajadores, honrados, leales, capaces de surgir solos, desean progresar, no borrachos ${ }^{2}$, aman la libertad, quieren ser útiles a Chile, patriotas y quieren educarse. El rótulo indios que desean ser chilenos fue empleado para nominar al segundo estereotipo, el cual incluyó los atributos positivos quieren ser útiles a Chile, desean ser chilenos, quieren educarse, patriotas, necesitan nuestra educación y desean progresar. Este estereotipo asigna a los mapuches la necesidad, y también el deseo, de desarrollo social y económico a través de una incorporación plena a la cultura mayoritaria, principalmente a través de la educación formal. El tercer estereotipo, llamado indios flojos, conflictivos y borrachos refleja una imagen desfavorable de los mapuches como personas alcohólicas, beligerantes e improductivas. Cinco atributos componen este estereotipo: borrachos, porfiados, violentos, deshonestos y flojos. Finalmente, el cuarto estereotipo ligado a los mapuches contemporáneos fue denominado gente silenciosa y desconfiada, ya que refleja una imagen asocial del mapuche. Este estereotipo incluye los atributos negativos callados y desconfiados.

En general, estos nueve estereotipos presentaron relativamente altos niveles de notoriedad indicando que, desde la perspectiva de los estudiantes, los estereotipos eran bastante generalizables entre los mapuches. Además, los estereotipos tendieron a ser

\footnotetext{
2 La expresión "no borrachos" y otras análogas indican que el atributo presentó una carga negativa en el factor.
} 
uniformes, señalando un sustancial consenso entre los estudiantes en la atribución de los mismos.

\section{Nuevo Escenario Intergrupal}

Desde el estudio realizado por Saiz (1991) hacia el término de los años 80 , al menos dos nuevos procesos sociales han contribuido al deterioro de las relaciones entre mapuches y chilenos no indígenas. En consonancia con el movimiento de emergencia indígena en Latinoamérica (Bengoa, 2000), en Chile ha ocurrido una agudización del conflicto histórico sobre derechos, tierras y recursos que mantienen, por una parte, sectores mapuches y, por otra, el Estado y algunos segmentos de la sociedad mayoritaria, hoy principalmente ligados a empresas agrícolas, forestales e hidroeléctricas (Saavedra, 2002). Además, el Estado ha generado un debate público sobre las relaciones históricas con los grupos originarios y sus consecuencias (e.g., Yáñez \& Aylwin, 2007) y ha desarrollado acciones restitutorias y reparadoras que, debido a la exclusión que conlleva la discriminación positiva, han generado rechazo y resentimiento en sectores no indígenas.

Considerando este nuevo escenario intergrupal, cabe preguntarse: ¿se mantienen o no vigentes en la actualidad los nueve estereotipos encontrados por Saiz (1991)? y ¿habrán surgido estereotipos nuevos? Este estudio propone respuestas afirmativas a estas preguntas y plantea que: (a) los estereotipos pueden permanecer inalterados, debido al modo en que se organizan cognitivamente sus contenidos, siendo difícilmente afectados por modificaciones contextuales y (b) la aparición de nuevos estereotipos puede derivar de cambios en las relaciones intergrupales. A continuación se exponen antecedentes teóricos y empíricos que apoyan estos planteamientos.

\section{Antecedentes Teóricos y Empíricos}

\section{Permanencia de los Estereotipos}

El estudio de Saiz (1991) reveló la presencia de múltiples estereotipos, algunos favorables y otros desfavorables, asociados a cada una de las dos categorías cronológicas de mapuches. Este hallazgo es convergente con la noción que los contenidos de los estereotipos se organizan en estructuras jerárquicas que reflejan imágenes asociadas a distintas subcate- gorías del exogrupo (Richards \& Hewstone, 2001). Al parecer, en la sociedad mayoritaria la categoría general mapuche incluye al menos dos subcategorías primarias (mapuche pretérito y mapuche contemporáneo) y cada una de ellas contiene un número de categorías secundarias subordinadas, aun más diferenciadas y menos incluyentes, asociadas a los distintos estereotipos.

¿Cómo generan los miembros del endogrupo esta subdivisión del exogrupo, especialmente frente a la incongruencia cognitiva que resulta de la coexistencia de imágenes favorables y desfavorables sobre el mismo exogrupo? Dos procesos de categorización parecen intervenir: la creación de subtipos (subtyping) y de subgrupos (subgrouping) (McCabe \& Brannon, 2004; Richards \& Hewstone, 2001). En el primer proceso, al enfrentar a miembros del exogrupo que son discordantes con los estereotipos previos, los integrantes del endogrupo reaccionan tratándolos como excepciones y clasificándolos en una subcategoría minoritaria separada de la categoría mayoritaria compuesta por los miembros que sí confirman los estereotipos (subtipos). Así, un subtipo representa a un conjunto de casos atípicos del exogrupo al cual se le asigna otro estereotipo. Un rasgo esencial de los subtipos es que la exclusión de los casos excepcionales fuera de los límites del exogrupo mayoritario permite mantener intactos los estereotipos previos (Hewstone, 1994). Por otra parte, en el segundo proceso, los integrantes del endogrupo separan a los miembros del exogrupo en varias subcategorías (subgrupos) sobre la base de semejanzas al interior de las subcategorías y de diferencias entre las subcategorías. En contraste con los subtipos, los miembros de los subgrupos no son percibidos como elementos que se desvían de la categoría mayoritaria. Por el contrario, los subgrupos son percibidos como compartiendo muchos de los atributos de la categoría superior aunque, en cada subgrupo, de una manera ligeramente diferente (Park, Ryan \& Judd, 1992).

El hallazgo de múltiples estereotipos, tanto positivos como negativos, asociados a los mapuches sugiere la elaboración cognitiva de subtipos y subgrupos por parte del endogrupo para resolver, respectivamente, la presencia de información discordante (estereotipos de valencia contraria asociados a una misma subcategoría) y la percepción de variabilidad dentro del exogrupo (estereotipos de igual valencia dentro una misma subcategoría). La simultaneidad de subtipos y subgrupos en cada subcategoría primaria de mapuche conduciría a la 
conservación de estereotipos de signo opuesto y, entre los estereotipos de signo compatible, llevaría al mantenimiento de su diferenciación interna. Así, en definitiva, la acción preservadora de estos dos procesos de categorización afectaría al conjunto de estereotipos, sean favorables o desfavorables, asociados a cada subcategoría primaria de mapuche. Esta línea de razonamiento lleva a esperar que los nueve estereotipos encontrados por Saiz (1991) continúen vigentes en la actualidad.

El planteamiento anterior es congruente, además, con antecedentes proporcionados por estudios recientes (Merino, Millamán, Quilaqueo \& Pilleux, 2004; Merino \& Quilaqueo, 2003; Quilaqueo \& Merino, 2003; Rojas \& Sepúlveda, 2002) que revelan la permanencia de varios de los estereotipos verificados por Saiz (1991). En esta misma línea, la enseñanza oficial de la historia nacional y los medios de comunicación social parecen estimular el aprendizaje y conservación de estos estereotipos (Casner, Navarrete, Rifo \& Zañartu, 2004; Pilleux, 2002; Quilaqueo \& Merino, 2003).

\section{Surgimiento de Nuevos Estereotipos}

La teoría del conflicto realista (Campbell, 1965; Olzak, 1992) contribuye a explicar la aparición de nuevos estereotipos. Un planteamiento central es que las actitudes y conductas tienden a reflejar los intereses de cada grupo. Cuando estos intereses son incompatibles y se compite por recursos escasos, inevitablemente surgen varias formas de rechazo grupal, entre otras, estereotipos negativos sobre el otro grupo (LeVine \& Campbell, 1972; Sherif, 1966). La competición puede incluir también pugna por estatus o poder. El surgimiento de nuevos estereotipos parece responder a modificaciones en las relaciones económicas o políticas entre los grupos que compiten (Bar-Tal \& Labin, 2001; Haslam, Turner, Oakes \& McGarty, 1992).

Indicios de nuevos estereotipos mapuches.Una revisión de la literatura reciente sugiere el surgimiento de dos nuevos estereotipos, ambos desfavorables, sobre los actuales mapuches. Un estereotipo adjudicaría atributos como subversivos, extremistas, prepotentes, que no miden riegos, violentos, peleadores, destructivos, traicioneros, enceguecidos (Amolef, 2004; Merino et al., 2004; Merino \& Quilaqueo, 2003; Quilaqueo \& Merino, 2003). Esta imagen negativa formaría parte del discurso de justificación de ciertos sectores de la sociedad mayoritaria que se muestran contrarios a las acciones reivindicativas de algunas organizaciones mapuches, atribuyendo a los propios indígenas la responsabilidad por la pobreza y falta de inversión en la región (Merino et al., 2004). Este estereotipo podría ser rotulado como terroristas.

El otro nuevo estereotipo sugerido por la literatura alude a medidas estatales de discriminación positiva hacia los mapuches, principalmente entrega de tierras y de recursos económicos, acciones que son percibidas como inmerecidas, y por tanto injustas, por sectores no indígenas (Merino et al., 2004; Merino \& Quilaqueo, 2003). Estas acciones de discriminación positiva, argumentan estos sectores, constituyen un error ya que las demandas por tierras carecerían de fundamento legal, los mapuches no contarían con el suficiente conocimiento para aprovechar adecuadamente los recursos entregados y no se les exigirían responsabilidades por los resultados, en circunstancias que el Estado sí exige responsabilidades a las personas no indígenas. Este nuevo estereotipo podría denominarse injustamente privilegiados. Reforzando los antecedentes expuestos, Casner et al. (2004) y Saiz, Merino y Quilaqueo (en prensa) documentan que estos nuevos estereotipos son efectivamente percibidos como tales por los propios mapuches.

Conflicto y nuevos estereotipos mapuches.Los dos estereotipos propuestos habrían surgido en razón de cambios en las relaciones entre endogrupo y exogrupo, acompañados por una intensificación de la competencia intergrupal, como plantea la teoría del conflicto realista. En particular, la imagen de terroristas emergería principalmente ligada a la reedición de demandas etnonacionales (Foerster \& Vergara, 2003; Saavedra, 2002) hechas por mapuches al Estado chileno, las cuales incluyen, entre otras aspiraciones, autonomía política y territorial (Marimán, Canuiqueo, Millalén \& Levil, 2006; Naguil, 1999). Estas demandas, que no han encontrado eco en ningún sector relevante de la sociedad mayoritaria, han sido a menudo apoyadas por acciones de fuerza (e.g., tomas de terrenos, obstrucción de caminos, incendios forestales y de inmuebles, destrucción de maquinaria agrícola y de transporte) en algunos sectores mapuches (Saavedra, 2002). Aunque no puede afirmarse que esta forma radical de postura etnonacional es generalizada entre los mapuches 
(ver, por ejemplo, von Baer, 2004), la capacidad de movilización de quienes la suscriben y la, a veces, vehemente respuesta en contrario del Estado, empresas privadas y propietarios de tierras no solo han intensificado el conflicto histórico sino que se han abierto nuevas áreas de disputas. En términos de la teoría del conflicto realista, mapuches y no indígenas no compiten ahora exclusivamente por recursos materiales sino, también, por estatus y poder. La imagen de terroristas constituiría para el endogrupo una adecuada explicación del cambio en las relaciones con los mapuches y, además, una justificación de su oposición a las reivindicaciones mapuches, en especial a las demandas etnonacionales.

Por su parte, la imagen de injustamente privilegiados derivaría de los intentos del Estado para remediar situaciones de inequidad histórica y/o actual de los mapuches, en respuesta a lo que Foerster y Vergara (2003) denominan reivindicación étnica de los mapuches. Estas acciones de discriminación positiva, que incluyen la entrega de recursos en condición de exclusividad a los mapuches, habría derivado en la emergencia del fenómeno de discriminación reversa (Dovidio \& Gaertner, 1998) en sectores no indígenas que comparten con los mapuches las mismas necesidades de tales recursos (Merino \& Quilaqueo, 2003). La discriminación reversa consideraría que el endogrupo resulta, a la postre, perjudicado ya que el trato preferente dado por el Estado a los mapuches viola creencias fundamentales acerca de una distribución justa de recursos basada en el mérito y no en la mera pertenencia étnica. En consonancia con la teoría del conflicto realista, la intervención reparadora del Estado chileno habría cambiado las relaciones entre endogrupo y exogrupo agudizando, en condiciones injustas, la competición entre ambos grupos por la obtención de recursos. Así, la imagen de injustamente privilegiados parece responder a un intento del endogrupo para explicar la arbitrariedad que percibe en el trato preferente dado a los mapuches y, a su vez, justificar su discrepancia con estas acciones estatales.

La teoría del conflicto realista y la literatura revisada permiten esperar la presencia en el endogrupo de estos dos nuevos estereotipos sobre los mapuches coetáneos. De ser esto efectivo, la inclusión de estos dos estereotipos negativos en el conjunto de estereotipos previos debería resolverse mediante la elaboración de nuevos subtipos y subgrupos.

En suma, el presente estudio se propuso evaluar: (1) la vigencia actual de los estereotipos encontrados por Saiz (1991) y (2) el surgimiento de dos nuevos estereotipos. Esta evaluación consideró el contenido, favorabilidad, notoriedad y uniformidad de los estereotipos.

\section{Método}

\section{Participantes}

Se empleó una muestra intencionada compuesta por 407 alumnos regulares de pregrado de la UFRO, Temuco. La selección de los participantes fue guiada de tal modo que esta muestra fuera equivalente, en cuatro variables relevantes, a aquella extraída en 1988 por Saiz (1991) desde esta misma universidad. Así, ambas muestras incluyeron solo estudiantes chilenos no indígenas que estaban cursando el primer o segundo año en sus respectivas carreras. Además, las muestras no difirieron significativamente según edad $(M=18,60, D E=1,21$ en $1988 ; M=18,73$, $D E=1,24$ en la presente muestra), $t(605)=-1,23, p$ $=0,22$, ni género $(48 \%$ de hombres en 1988 y $53 \%$ en la presente muestra), $z=-1,18, p=0,24$. Finalmente, al igual que en la muestra de Saiz (1991), el presente estudio incluyó una amplia variedad de carreras $(n=20)$. La distribución porcentual de los participantes según las cuatro áreas disciplinarias de pregrado de la universidad fue muy similar a la distribución poblacional, siendo los respectivos porcentajes: ingeniería ( $38 \%$ vs. $43 \%$ ), salud ( $25 \%$ vs. $24 \%)$, educación y ciencias sociales $(24 \% v s$. $26 \%$ ) y agricultura ( $13 \%$ vs. $7 \%$ ). Casi todos los participantes tenían residencia urbana previa al ingreso a la universidad (86\%) y procedían de las regiones del Bío Bío, de La Araucanía, de Los Ríos y de Los Lagos (88\%), regiones que incluyen al 59\% de la población mapuche nacional (Instituto Nacional de Estadística, 2005). En estas regiones, precisamente, la agudización del conflicto ha alcanzado mayor virulencia y se han concentrado las acciones estatales de discriminación positiva.

\section{Instrumento}

Las tres primeras secciones de un cuestionario de construcción ad-hoc recogieron información pertinente al presente estudio. En la primera sección se indicó el propósito del estudio, se proporcionaron las instrucciones generales y se incluyeron ítems sobre información sociodemográfica del partici- 
pante, siendo los relevantes: género, edad, carrera, nivel cursando en la carrera, residencia familiar rural-urbana, región de procedencia y pertenencia a grupos indígenas chilenos.

En la segunda sección se presentó el estímulo mapuches del pasado seguido de una lista de 18 atributos. En la tercera sección se exhibió el estímulo mapuches del presente seguida de otra lista de 30 atributos. Los atributos de cada lista fueron aquellos que integran, respectivamente, los estereotipos sobre los mapuches pretéritos (15 atributos) y contemporáneos (18 atributos) encontrados por Saiz (1991). La segunda lista incluyó, además, atributos que procuran reflejar los dos nuevos estereotipos sobre mapuches sugeridos por la literatura, esto es, terroristas (5 atributos: agresivos, extremistas, destructivos, conflictivos, prepotentes) e injustamente privilegiados (4 atributos: aprovechadores, manipuladores, oportunistas, desvergonzados). Los atributos de estos dos últimos estereotipos fueron aquellos más frecuentemente mencionados por una muestra accidental de 42 alumnos de la carrera de Psicología de la UFRO, a quienes se les solicitó describir, por escrito, a una "persona terrorista" o una "persona injustamente privilegiada". Finalmente, cada lista incluyó, como distractores, tres atributos no asociados a estereotipos sobre los mapuches (distraídos, ansiosos, alegres). En cada lista, los adjetivos fueron distribuidos aleatoriamente.

En la segunda y tercera secciones se pidió a los participantes que opinaran sobre cuántos de los mapuches señalados por cada estímulo tenían (en el caso de mapuche del pasado) o tienen (en el caso de mapuche del presente) cada atributo. Los participantes respondieron, frente a cada atributo, seleccionando una de siete opciones que cubrían desde ninguno (codificada luego con 1 punto) hasta todos (7 puntos), con una opción central rotulada como la mitad (4 puntos).

\section{Procedimiento}

Los participantes respondieron, de manera voluntaria, anónima y sin límites de tiempo, el cuestionario al inicio o al final de una clase regular, contando con la autorización previa del profesor de la asignatura. La administración del cuestionario fue realizada por una alumna del programa de Magíster en Psicología de la UFRO y la actividad fue presentada como parte de su proyecto de tesis. Antes de la entrega de los cuestionarios, cada participante leyó y completó un formulario de consentimiento informado. Su participación fue retribuida con una barra de confites. La administración del cuestionario fue realizada durante 2006 .

\section{Plan de Análisis}

Para identificar los contenidos de los estereotipos, las respuestas individuales de asignación de atributos fueron divididas en tres conjuntos: (1) aquellas referidas a los mapuches pretéritos (15 atributos) y, entre las respuestas vinculadas a los mapuches contemporáneos; (2) aquellas referidas a los atributos incluidos en los estereotipos reportados por Saiz (1991) (18 atributos); y (3) aquellas referidas a los dos nuevos estereotipos propuestos (9 atributos). Cada conjunto de respuestas fue sometido a un análisis factorial mediante extracción por ejes principales y rotación tanto ortogonal ( $v a-$ rimax) como oblicua (direct oblimin). La solución factorial fue seleccionada usando como criterios los autovalores, el gráfico de sedimentación y las cargas factoriales. Los factores fueron definidos por atributos con cargas iguales o mayores a $0,30 .^{3}$

En la interpretación del contenido de los dos primeros conjuntos de factores se examinó si cada factor replicaba alguno de los estereotipos encontrados por Saiz (1991). Este examen fue realizado mediante el cálculo del índice de similitud destacada s (Cattel, Balcar, Horn \& Nesselroade, 1969), una medida de semejanza estructural de las cargas factoriales entre pares de factores obtenidos con las mismas variables pero en muestras independientes de participantes (Tabachnick \& Fidell, 1989). Usando un valor absoluto de 0,30 para considerar una carga factorial como destacada, el índice $s$ comparó los factores que parecían ser inicialmente similares a uno o algunos de los estereotipos (factores) reportados por Saiz (1991), atendiendo al número de atributos comunes. Se considera que un índice $s \geq 0,85$ refleja identidad entre los factores (ten Berge, 1977). En la interpretación del contenido del tercer conjunto de factores se inspeccionó si sus atributos reflejaban los dos nuevos estereotipos propuestos.

Luego de interpretados los factores, se computó el promedio de las respuestas dadas por cada participante en los atributos que definen cada factor.

\footnotetext{
Todos los análisis factoriales posteriores fueron ejecutados siguiendo estos mismos procedimientos de extracción y rotación.
} 
Este promedio indica las diferencias individuales en el grado en que cada participante suscribe cada estereotipo (puntaje de estereotipia).

Finalmente, se obtuvieron puntajes de favorabilidad, notoriedad y uniformidad para cada uno de los atributos no redundantes de los estereotipos que surgieron en los análisis factoriales. Una muestra adicional compuesta por 106 alumnos regulares de primer o segundo año de la carrera de Psicología de la UFRO evaluaron la favorabilidad de estos atributos, empleando una escala de respuesta de cinco opciones que iba desde muy negativo (1 punto) hasta muy positivo (5 puntos). Luego, se computó una media de favorabilidad para cada atributo. Elevados puntajes de favorabilidad indican que el atributo es juzgado como teniendo una alto grado de deseabilidad social. Por otra parte, las respuestas dadas por los participantes del presente estudio al adscribir atributos fueron usadas para computar tanto un puntaje de notoriedad, reflejado por el promedio de suscripción del atributo, como un puntaje de uniformidad, reflejado por el valor inverso de la desviación típica de la suscripción del atributo. Elevados puntajes de notoriedad indican que un mayor número de mapuches es percibido como poseyendo esos atributos, mientras que elevados puntajes de uniformidad señalan mayores grados de consenso entre los estudiantes sobre la asignación de esos atributos a los mapuches. Con la excepción de los puntajes de uniformidad, los puntajes de favorabilidad y notoriedad fueron invertidos para aquellos atributos con cargas negativas en un factor. Como en Saiz (1991), para facilitar la comparación de los estereotipos los puntajes de notoriedad (en una escala de 1 a 7 puntos) y uniformidad (en una escala de 0 a 3 puntos) fueron linealmente transformados (Aiken, 1987) a la misma escala de 1 a 5 puntos de los puntajes de favorabilidad.

\section{Resultados}

Los resultados son presentados en dos secciones. En la primera se reporta el contenido de los estereotipos asociados a los mapuches pretéritos y contemporáneos, diferenciando entre estos últimos aquellos referidos a los estereotipos encontrados por Saiz (1991) y aquellos propuestos como nuevos estereotipos. En la segunda sección se reporta la favorabilidad, notoriedad y uniformidad de los estereotipos.

\section{Contenido de los Estereotipos}

Se retuvieron tres factores tanto en el primer como en el segundo conjunto de respuestas, y dos factores en el tercer conjunto, soluciones que explican el $53,8 \%, 49,7 \%$ y $62,2 \%$ de la varianza común de los atributos, respectivamente. Al estar los factores de cada conjunto moderada o altamente correlacionados, se interpretaron las soluciones oblicuas.

Estereotipos adscritos a los mapuches pretéritos. En la Tabla 1 se presenta la estructura trifactorial que adoptan los atributos asociados a los mapuches del pasado. Tres atributos (no querían ser chilenos, expresivos, no querían educarse) no presentaron cargas suficientemente altas en ningún factor. El Factor 1 tuvo una correlación de $r=-0,13$ con el Factor 2 y $r=0,62$ con el Factor 3, en tanto que el Factor 2 tuvo una correlación de $r=0,23$ con el Factor 3 (en todos, $p<0,01$ ).

Cuatro de los cinco atributos que definen el Factor 1 (responsables, leales, honrados, inteligentes) son también los atributos que definen el estereotipo gente noble reportado por Saiz (1991). El índice $s=$ $0,89, p<0,001$ señala una equivalencia entre estos factores. Además, el Factor 1 posee una moderada semejanza con el estereotipo previo indios industriosos, $s=0,57, p<0,05$. De hecho, los atributos trabajadores y responsables del factor corresponden a dos de los tres atributos que definen a este estereotipo. El Factor 1, entonces, parece contener una fusión de dos estereotipos previos, razón por la cual fue denominado gente noble e industriosa. Este factor reflejaría una percepción de los mapuches pretéritos como personas dignas, confiables y hacendosas.

El Factor 2 coincide exactamente con el estereotipo indios hostiles (Saiz, 1991), $s=1,00, p<$ 0,001 . En efecto, los tres atributos que definen el Factor 2 (porfiados, violentos, desconfiados) definen también ese estereotipo. Al constituir una réplica cabal, este factor fue denominado con el mismo rótulo previo. Finalmente, todos los atributos que componen el Factor 3 (fuertes, valientes, rápidos, amaban la libertad, inteligentes) forman también parte del estereotipo valientes guerreros (Saiz, 1991). La única discrepancia fue el atributo leales del estereotipo que no apareció integrando el Factor 3. Pese a ello, el índice $s=0,91, \mathrm{p}<0,001$ indica identidad factorial y, por tanto, el factor obtenido fue denominado valientes guerreros. 
Tabla 1

Análisis Factorial de Atributos Adscritos a los Mapuches Pretéritos: Réplica de los Estereotipos Encontrados por Saiz (1991)

\begin{tabular}{lccc}
\hline \multirow{2}{*}{ Rótulos de factores y atributos } & \multicolumn{3}{c}{ Factores } \\
\cline { 2 - 4 } Gente noble e industriosa & \multicolumn{3}{c}{2} \\
$\quad$ Responsables & $\mathbf{0 , 8 9}$ & $-0,04$ & $-0,12$ \\
Leales & $\mathbf{0 , 6 7}$ & 0,04 & 0,07 \\
Honrados & $\mathbf{0 , 5 3}$ & 0,06 & 0,07 \\
Trabajadores & $\mathbf{0 , 4 6}$ & $-0,11$ & 0,16 \\
Inteligentes & $\mathbf{0 , 3 9}$ & $-0,11$ & $\mathbf{0 , 3 0}$ \\
Indios hostiles & & & \\
Porfiados & 0,01 & $\mathbf{0 , 7 5}$ & $-0,09$ \\
Violentos & $-0,13$ & $\mathbf{0 , 5 0}$ & 0,14 \\
Desconfiados & 0,05 & $\mathbf{0 , 4 3}$ & 0,01 \\
Valientes guerreros & & & \\
Fuertes & $-0,09$ & $-0,05$ & $\mathbf{0 , 8 1}$ \\
Valientes & 0,14 & 0,00 & $\mathbf{0 , 5 6}$ \\
Rápidos & 0,15 & 0,10 & $\mathbf{0 , 4 5}$ \\
Amaban la libertad & 0,07 & 0,09 & $\mathbf{0 , 3 1}$ \\
\hline Autovalores & 3,7 & 1,8 & 1,2 \\
\% de varianza & 30,7 & 15,3 & 7,8 \\
\hline
\end{tabular}

Nota. Las cargas de los atributos que definen cada factor se destacan en negrita.

En suma, en relación a los mapuches pretéritos, cuatro de los cinco estereotipos reportados por Saiz (1991) resultaron replicados; la excepción fue indios que resistían la chilenización. Estos hallazgos tienden a apoyar, como se esperaba, la vigencia de estos estereotipos.

Para indagar la estructura más inclusiva que adoptan estos tres estereotipos, los correspondientes puntajes de estereotipia fueron examinados mediante un análisis factorial de segundo orden. Previamente, la consistencia interna (alfa de Cronbach) de estos puntajes fue satisfactoria, en particular si se atiende a la baja cantidad de ítems (atributos) que los componen $(\alpha=0,78$ para gente noble e industrios $a, \alpha=$ 0,57 para indios hostiles y $\alpha=0,70$ para valientes guerreros). Emergió una estructura compuesta por dos factores independientes $(r=-0,02, p>0,05)$ que explican el $90,4 \%$ de la varianza común de estos estereotipos. El primer factor (autovalor $=1,7$, varianza explicada $=55,9 \%$ ) incluyó los estereotipos positivos valientes guerreros y gente noble e industriosa, ambos con una carga de 0,85 . El estereotipo negativo, indios hostiles, integró el otro factor (autovalor $=1,1$, varianza explicada $=$ $34,4 \%$ ) con una carga de 0,49 .
Estereotipos adscritos a los mapuches contemporáneos. En la Tabla 2 se exhibe la configuración trifactorial de los atributos referidos a los estereotipos reportados por Saiz (1991) sobre los mapuches del presente. Solo el atributo callados careció de una carga suficientemente alta en algún factor. El Factor 1 tuvo una correlación de $r=-0,18$ con el Factor 2 y de $r=0,40$ con el Factor 3, mientras que el Factor 2 tuvo una correlación de $r=-0,15$ con el Factor 3 (todos con $p<0,01$ ).

Diez de los once atributos que definen el Factor 1 (inteligentes, responsables, leales, capaces de surgir solos, trabajadores, honrados, desean progresar, quieren educarse, aman la libertad, no borrachos) definen también el estereotipo buenos ciudadanos (Saiz, 1991). Además, este factor incluye el atributo pacíficos, que no forma parte del estereotipo original $\mathrm{y}$, a su vez, no incluye los dos atributos (quieren ser útiles a Chile, patriotas) que integran este estereotipo. Pese a estas discrepancias, el índice $s=0,87$, $p<0,001$ indica equivalencia factorial, razón por la cual este factor también fue denominado buenos ciudadanos.

El Factor 2 es moderadamente semejante al estereotipo indios flojos, conflictivos y borrachos 
Tabla 2

Análisis Factorial de Atributos Adscritos a los Mapuches Contemporáneos: Réplica de los Estereotipos Encontrados por Saiz (1991)

\begin{tabular}{lrrr}
\hline \multirow{2}{*}{ Rótulos de factores y atributos } & \multicolumn{3}{c}{ Factores } \\
\cline { 2 - 4 } & \multicolumn{3}{c}{2} \\
\hline Buenos ciudadanos & $\mathbf{0 , 7 2}$ & 0,04 & $-0,06$ \\
Inteligentes & $\mathbf{0 , 6 7}$ & $-0,16$ & 0,10 \\
Responsables & $\mathbf{0 , 6 4}$ & 0,00 & 0,01 \\
Leales & $\mathbf{0 , 6 1}$ & $-0,16$ & $-0,08$ \\
Capaces de surgir solos & $\mathbf{0 , 6 0}$ & 0,03 & 0,15 \\
Trabajadores & $\mathbf{0 , 5 8}$ & $-0,14$ & 0,05 \\
Honrados & $\mathbf{0 , 5 5}$ & 0,02 & 0,15 \\
Desean progresar & $\mathbf{0 , 4 2}$ & $-0,05$ & $\mathbf{0 , 3 6}$ \\
Quieren educarse & $\mathbf{0 , 3 3}$ & 0,22 & $-0,03$ \\
Aman la libertad & & & \\
Antisociales e incultos & $-0,10$ & $\mathbf{0 , 7 0}$ & $-0,14$ \\
Porfiados & 0,07 & $\mathbf{0 , 5 6}$ & $-0,14$ \\
Desconfiados & $\mathbf{0 , 3 3}$ & $\mathbf{0 , 4 1}$ & $-0,04$ \\
Borrachos & $\mathbf{0 , 3 3}$ & $\mathbf{- 0 , 3 6}$ & 0,19 \\
Pacíficos & $-0,03$ & $\mathbf{0 , 3 4}$ & 0,26 \\
Necesitan nuestra educación & & & \\
Indios que desean ser chilenos & $-0,06$ & $-0,10$ & $\mathbf{0 , 6 8}$ \\
Desean ser chilenos & 0,26 & 0,00 & 0,64 \\
Quieren ser útiles a Chile & 0,07 & $-0,03$ & 0,35 \\
Patriotas & 5,4 & 1,7 & 1,4 \\
\hline Autovalores & 31,5 & 10,1 & 8,1 \\
\% de varianza & & & \\
\hline
\end{tabular}

Nota. Las cargas de los atributos que definen cada factor se destacan en negrita.

(Saiz, 1991), $s=0,60, p<0,01$. Aunque el factor incluye la caracterización de antisociales (porfiados, borrachos, no pacificos) contendida en el estereotipo previo, caracterización que es remarcada por la adición de un rasgo de suspicacia (desconfiados), se pierde la visión original de perezosos y deshonestos (no trabajadores, no honrados) del estereotipo. Esta caracterización de antisociales es matizada, además, por una imagen de personas ineducadas (necesitan nuestra educación). El rótulo antisociales e incultos fue usado para denominar este factor.

En el Factor 3, si bien sus cuatro atributos (desean ser chilenos, quieren ser útiles a Chile, patriotas, quieren educarse) forman también parte del estereotipo indios que desean ser chilenos (Saiz 1991), no se incluyen dos atributos (desean progresar, necesitan nuestra educación) presentes en el estereotipo. Con todo, este factor puede ser considerado equivalente a este estereotipo, $s=0,85$, $p=0,001$, ya que captura la misma percepción estereotipada original que asigna a los mapuches coetáneos la aspiración a una identidad chilena y a una incorporación plena a la sociedad mayoritaria, principalmente a través de la educación. Por tal razón, este factor conservó el mismo rótulo del estereotipo original.

Sintetizando, en relación a los mapuches coetáneos, dos de los cuatro estereotipos reportados por Saiz (1991) resultaron plenamente replicados mientras que un tercero alcanzó una réplica parcial. Solo indios que resistían la chilenización no fue confirmado. Al igual como ocurrió respecto de los mapuches pretéritos, estos resultados tienden a respaldar la continuidad temporal de estos estereotipos.

En la Tabla 3 se presenta la estructura bifactorial de los atributos postulados para reflejar los nuevos estereotipos. Los factores presentaron una correlación de $r=0,75(p<0,001)$ y todos los atributos obtuvieron cargas factoriales suficientemente altas en al menos un factor. El Factor 1 incluye los cuatro atributos (aprovechadores, oportunistas, manipuladores, desvergonzados) destinados a medir el estereotipo injustamente privilegiados, con la adición inesperada de un atributo (prepotentes) dirigido 
Tabla 3

Análisis Factorial de Atributos Adscritos a los Mapuches Contemporáneos: Examen de los Dos Nuevos Estereotipos Propuestos

\begin{tabular}{lcc}
\hline \multirow{2}{*}{ Rótulos de factores y atributos } & \multicolumn{2}{c}{ Factores } \\
\cline { 2 - 3 } & 1 & 2 \\
\hline Injustamente privilegiados & $\mathbf{0 , 7 4}$ & 0,02 \\
Aprovechadores & $\mathbf{0 , 7 2}$ & $-0,02$ \\
Oportunistas & $\mathbf{0 , 6 7}$ & 0,01 \\
Manipuladores & $\mathbf{0 , 5 1}$ & 0,04 \\
Desvergonzados & & \\
Terroristas & $-0,02$ & $\mathbf{0 , 9 0}$ \\
Conflictivos & $-0,09$ & $\mathbf{0 , 7 2}$ \\
Extremistas & 0,18 & $\mathbf{0 , 5 9}$ \\
Destructivos & 0,25 & $\mathbf{0 , 5 1}$ \\
Agresivos & $\mathbf{0 , 3 2}$ & $\mathbf{0 , 4 8}$ \\
$\quad$ Prepotentes & 4,7 & 1,1 \\
\hline Autovalores & 51,9 & 10,3 \\
\% de varianza & & \\
\hline
\end{tabular}

Nota. Las cargas de los atributos que definen cada factor se destacan en negrita.

originalmente a medir el otro estereotipo. Esta adición, sin embargo, es plenamente convergente con el contenido sugerido para este estereotipo. El Factor 2, por su parte, incluye a los cinco atributos (conflictivos, extremistas, destructivos, agresivos, prepotentes) destinados a evaluar el estereotipo terroristas. Esta configuración bifactorial respalda plenamente la existencia de los dos nuevos estereotipos, como se esperaba.

Al igual como se procedió con los estereotipos que emergieron asociados a los mapuches pretéritos, mediante un análisis factorial de segundo orden se indagó la estructura más inclusiva que adoptan los cinco estereotipos sobre los mapuches coetáneos. Sus correspondientes puntajes de estereotipia obtuvieron una adecuada consistencia interna según alfa de Cronbach: buenos ciudadanos $(\alpha=0,85)$, antisociales e incultos $(\alpha=0,69)$, indios que desean ser chilenos $(\alpha=0,68)$, injustamente privilegiados $(\alpha=0,81)$ y terroristas $(\alpha=0,86)$. El análisis reveló una configuración de dos factores relacionados inversamente $(r=-0,62, p<0,001)$ que dan cuenta de un $82,3 \%$ de la varianza de los estereotipos. El primer factor (autovalor $=3,2$, varianza explicada $=$ $63,8 \%$ ) incluyó los estereotipos terroristas (con una carga de 0,90), injustamente privilegiados $(0,77)$ y antisociales e incultos $(0,77)$, todos negativos. El segundo factor (autovalor $=1,2$, varianza explicada $=18,6 \%$ ) incorporó a los dos estereotipos positivos: buenos ciudadanos $(0,96)$ e indios que desean ser chilenos $(0,60)$.

\section{Favorabilidad, Notoriedad y Uniformidad de los} Estereotipos

En la columnas 3, 5 y 7 de la Tabla 4 se presentan los promedios de favorabilidad, notoriedad y uniformidad obtenidos por cada estereotipo. Además, en las columnas 2, 4 y 6 de esa tabla se presentan los promedios obtenidos por Saiz (1991) en los estereotipos idénticos (homónimos) o similares a los del presente estudio. ${ }^{4}$ Una serie de 18 pruebas $t$ para muestras independientes reveló ausencia de diferencias significativas entre los respectivos promedios de favorabilidad, notoriedad y uniformidad de ambos estudios, indicando invarianza a través del tiempo.

Los estereotipos surgidos en esta investigación pueden ser descritos clasificando sus promedios en uno de cuatro intervalos: bajo $(1,0-1,9)$, moderadamente bajo $(2,0-2,9)$, moderadamente alto $(3,0$ - 3,9) y alto $(4,0-5,0)$. Mientras cuatro estereotipos (indios hostiles, antisociales e incultos, injustamente privilegiados, terroristas) fueron alta o moderadamente desfavorables, los cuatro estereotipos restantes fueron altamente favorables. Ningún estereotipo resultó ser ambivalente ya que los atributos que definen a cada factor fueron homogéneamente

\footnotetext{
4 Debe precisarse, para una mejor comprensión, que tanto en el presente estudio como en Saiz (1991) estos promedios fueron calculados considerando los atributos, y no los participantes, como las unidades de análisis.
} 
Tabla 4

Promedios de Favorabilidad, Notoriedad y Uniformidad de los Estereotipos Encontrados por Saiz (1991) (A) y por el Presente Estudio (B)

\begin{tabular}{|c|c|c|c|c|c|c|c|c|c|}
\hline \multirow{2}{*}{ Estereotipos } & \multicolumn{3}{|c|}{ Favorabilidad } & \multicolumn{3}{|c|}{ Notoriedad } & \multicolumn{3}{|c|}{ Uniformidad } \\
\hline & A & & B & A & & B & A & & B \\
\hline \multicolumn{10}{|l|}{ Mapuches pretéritos } \\
\hline Gente noble e industriosa & $4,48^{\mathrm{a}}$ & $=$ & 4,61 & $3,52^{\mathrm{a}}$ & $=$ & 3,49 & $2,99^{a}$ & $=$ & 3,02 \\
\hline Indios hostiles & 2,32 & $=$ & 2,25 & 4,02 & $=$ & 3,88 & 3,20 & $=$ & 3,08 \\
\hline Valientes guerreros & 3,94 & $=$ & 3,97 & 4,18 & $=$ & 3,96 & 3,59 & $=$ & 3,39 \\
\hline \multicolumn{10}{|l|}{ Mapuches contemporáneos } \\
\hline Buenos ciudadanos & 4,49 & $=$ & 4,50 & 3,44 & $=$ & 3,36 & 3,23 & $=$ & 3,13 \\
\hline Antisociales e incultos & $1,77^{\mathrm{b}}$ & $=$ & 2,62 & $2,90^{\mathrm{b}}$ & $=$ & 3,58 & $3,24^{\mathrm{b}}$ & $=$ & 3,04 \\
\hline Indios que desean ser chilenos & 4,37 & $=$ & 3,95 & 3,64 & $=$ & 3,05 & 2,32 & $=$ & 2,83 \\
\hline Injustamente privilegiados & -- & & 1,89 & -- & & 2,96 & -- & & 2,98 \\
\hline Terroristas & -- & & 1,60 & -- & & 3,27 & -- & & 2,95 \\
\hline
\end{tabular}

Nota. El signo $=$ indica que los promedios no difieren significativamente con $p<0,0027$, según ajuste de Bonferroni. Promedio de los estereotipos gente noble e indios industriosos (Saiz, 1991).

Promedio del estereotipo flojos, conflictivos y borrachos (Saiz, 1991).

favorables o desfavorables. En notoriedad todos los estereotipos presentaron niveles moderadamente altos. Esto significa que, desde la perspectiva de los participantes, los estereotipos parecen caracterizar a segmentos sustantivos de mapuches pretéritos y coetáneos. Finalmente, todos los estereotipos presentan niveles de uniformidad situados en torno al punto central de la escala $(3,0)$, sugiriendo un relativamente moderado nivel de acuerdo entre los estudiantes en la atribución de los estereotipos a los mapuches.

\section{Discusión}

Esta investigación se propuso indagar la vigencia actual de los nueve estereotipos sobre los mapuches reportados por Saiz (1991) y el surgimiento de dos nuevos estereotipos. En relación al primer objetivo, los resultados tendieron a confirmar la expectativa que los estereotipos, tanto aquellos asociados a los mapuches pretéritos como contemporáneos, se han mantenido relativamente invariables a través de 18 años. La vigencia no solo incluye la conservación de los contenidos sino, también, de los niveles de favorabilidad, notoriedad y uniformidad de los estereotipos. Además, estos hallazgos ratifican indicios reportados previamente (e.g., Merino et al., 2004) sobre la permanencia actual de algunos de estos estereotipos.
Dentro de este cumplimiento general de expectativas hubo, sin embargo, tres excepciones, todas referidas al contenido. Por una parte, el factor antisociales e incultos es similar, aunque no equivalente, al estereotipo anterior indios flojos, conflictivos y borrachos, ambos sobre los mapuches coetáneos. Esta similitud sugiere que el contenido del primero podría haber evolucionado a partir del segundo, eliminando o sustituyendo algunos atributos previos. La ausencia de diferencias significativas en la favorabilidad, notoriedad y uniformidad de estos dos estereotipos refuerza esta idea. En todo caso, esta eventual evolución queda abierta a verificación. Por otra parte, no se encontró evidencia sobre la permanencia de dos estereotipos previos asociados originalmente a los antiguos mapuches (indios que resistían la chilenización) y a los actuales mapuches (gente silenciosa $y$ desconfiada). Debe recordarse, sin embargo, que ambos estereotipos estaban definidos por solo dos atributos. Puesto que estas configuraciones mínimas suelen ser débiles e inestables, es probable que hayan dejado de ser creencias socialmente compartidas en esta población estudiantil.

En relación al segundo objetivo de este estudio, los resultados corroboran plenamente la expectativa derivada desde la teoría del conflicto realista (Campbell, 1965) sobre el surgimiento de dos nuevos estereotipos desfavorables que describen a los mapuches coetáneos como terroristas, uno, e injustamente privilegiados, el otro. Se ratifican, así, las presunciones sobre la formación de estos estereotipos (e.g., Amolef, 2004; Merino et al., 2004) 
y se subraya el rol que desempeña la alteración de las relaciones intergrupales, especialmente la agudización de un conflicto histórico y los intentos por contenerlo o resolverlo, como determinantes de la emergencia de estereotipos negativos.

Tres aspectos de estos resultados serán comentados. En primer lugar, más allá de la evidente interpretación de sus contenidos, los estereotipos encontrados parecen ser también psicosocialmente relevantes si se atiende a las intensidades que ellos exhiben en las restantes dimensiones. En términos de favorabilidad, los estereotipos tienden a agruparse claramente en positivos o negativos, como en Saiz (1991). Este resultado, junto a la ausencia de estereotipos ambivalentes, sugiere que estos estudiantes tienen imágenes favorables o desfavorables marcadamente definidas y diferenciadas sobre los mapuches. Todos los estereotipos presentaron niveles moderadamente altos de notoriedad, esto es, los estudiantes tienden a creer que los estereotipos son aplicables a sectores relativamente amplios de mapuches. Este hallazgo ilustra la sobregeneralización que haría el endogrupo al asignar estereotipos al exogrupo (Brigham, 1971). Los moderados niveles de uniformidad de los estereotipos indican un relativo consenso en las creencias estereotípicas dentro del endogrupo. Esto es importante ya que los estereotipos uniformes poseerían mayor significado social en el endogrupo y, por tanto, podrían predecir más acertadamente la interacción con el exogrupo (Saiz et al., en prensa).

En segundo lugar, la articulación que adoptan los estereotipos es congruente con procesos de organización cognitiva que resuelven la coexistencia de información contradictoria sobre un mismo exogrupo. Los estereotipos que emergieron asociados a cada categoría cronológica de mapuche configuran estructuras más inclusivas, de segundo orden, que conglomeran estereotipos de igual valencia, aunque de contenido diferente (subgrupos) $\mathrm{y}$, además, diferencian claramente estereotipos de valencia contrapuesta (subtipos). Como se señaló, estos dos procesos conducirían a la conservación de los estereotipos de valencia contrapuesta, al separarlos en categorías diferentes y, además, llevaría al mantenimiento de los estereotipos de igual valencia, al preservar sus diferenciaciones internas. Interesante es advertir que, una vez surgidos en razón de cambios contextuales, los nuevos estereotipos parecen integrarse coherentemente en la estructura de estereotipos precedentes. Debido a la acción preservadora de los subtipos y subgrupos, es dable hipotetizar que los nuevos estereotipos, al igual como ocurre con los estereotipos previos, tienden a persistir en el tiempo.

Si bien este estudio no demuestra la intervención de los mecanismos de subgrupos y subtipos, las estructuras resultantes concuerdan con lo que cabría esperar si estos mecanismos estuvieran, en realidad, operando. Aunque otros procesos cognitivos y psicosociales han sido propuestos para explicar la resistencia al cambio de los estereotipos (Schneider, 2005), el enfoque de subtipos y subgrupos parece ser más parsimonioso ya que permitiría explicar no solo la estabilidad temporal de los estereotipos a través del tiempo sino, también, la coexistencia en el endogrupo de estereotipos de valencia contraria. En cualquier caso, la intervención efectiva de estos mecanismos en la preservación de los estereotipos mapuches es un asunto que requiere investigación.

En tercer lugar, resulta interesante discutir qué consecuencias pudieran tener estos estereotipos, como creencias socialmente compartidas, para las relaciones intergrupos en el marco de un conflicto histórico, actualmente intensificado. Como se señaló, este conflicto no entraña hoy una contienda generalizada entre los dos grupos sino que contrapone a segmentos particulares de cada uno. Además, como puede inferirse de su estructura, los estereotipos parecen referirse a diferentes conjuntos de mapuches. Esta diferenciación interna del exogrupo sería menos relevante en el caso de los estereotipos sobre los mapuches pretéritos por cuanto estos, en tanto colectivo sin existencia contemporánea, constituyen para la sociedad mayoritaria un exogrupo puramente imaginado, con el cual es imposible la interacción. En razón de esta autonomía contextual, estos estereotipos no impactarían las relaciones grupales. Por otra parte, los efectos de los estereotipos asociados a los mapuches coetáneos dependerían de la valencia positiva o negativa de los atributos. Así, los estereotipos desfavorables solo contribuirían al deterioro de relaciones con mapuches que son percibidos como involucrados en el conflicto. De hecho, las imágenes de antisociales e incultos, injustamente privilegiados y terroristas parecen ajustarse bastante a esta percepción. Estos estereotipos negativos no tendrían, por tanto, consecuencias en las relaciones con mapuches juzgados como no implicados en el conflicto. El patrón inverso sería esperable en los estereotipos favorables. Así, las imágenes de buenos ciudadanos e indios que desean ser chilenos impactarían únicamente la interacción con mapuches percibidos como ajenos al conflicto. 
El presente estudio presenta algunas limitaciones. Cuando se comparan muestras independientes evaluadas en dos tiempos diferentes, un aspecto crítico para concluir estabilidad o cambio entre las dos mediciones es asegurar que las muestras sean equivalentes. Aunque la selección de los participantes procuró igualar ambas muestras en variables que parecieron relevantes, ello no asegura que la equivalencia haya sido completa. Una eventual falta de equivalencia habría contribuido a generar diferencias espurias entre las dos mediciones, confundiéndose con diferencias sustantivas. Sin embargo, la casi total ausencia de diferencias al contrastar ambas mediciones respalda la idea que las muestras eran razonablemente comparables.

Finalizando, debe considerarse que el presente estudio y el de Saiz (1991) fueron realizados en una población universitaria específica y, por tanto, sus resultados no pueden ser generalizados sin precaución a otras poblaciones de chilenos no indígenas. Muy probablemente, los estereotipos aquí obtenidos se superponen solo parcialmente con los estereotipos existentes en otras poblaciones. De hecho, la presencia de estereotipos tanto favorables como desfavorables asociados a los mapuches contemporáneos solo ha sido detectada, hasta ahora, en estudiantes universitarios (Saiz et al., en prensa). Igualmente, la evolución de los estereotipos aquí examinada no necesariamente ha de ser la misma en otros colectivos.

\section{Referencias}

Aiken, L. R. (1987). Formulas for equating ratings on different scales. Educational and Psychological Measurement, 47, 51-54.

Amolef, A. (2004). La alteridad en el discurso mediático: mapuches y la prensa chilena II. Boletín IFP, 1(7), 19-22.

Bar-Tal, D. \& Labin, D. (2001). The effect of a major event on stereotyping: Terrorist attacks in Israel and Israeli adolescents' perceptions of Palestinians, Jordanians, and Arabs. European Journal of Social Psychology, 31, 265-280.

Bengoa, J. (2000). La emergencia indígena en América Latina. Santiago, Chile: Fondo de Cultura Económica.

Brigham, J. C. (1971). Ethnic stereotypes. Psychological Bulletin, 76, 15-38.

Campbell, D. T. (1965). Ethnocentrism and other altruistic motives. En D. Levine (Ed.), Nebraska Symposium on Motivation (pp. 283-311). Lincoln, NE: University of Nebraska Press.

Casner, M. E., Navarrete, R. A., Rifo, H. J. \& Zañartu, N. L. (2004). La discriminación étnica como contexto obstaculizador del desarrollo humano: representaciones sociales de jóvenes mapuche en la ciudad de Temuco. Tesis no publicada para optar al grado de Licenciado en Psicología, Universidad de La Frontera, Temuco, Chile.

Cattel, R. B., Balcar, K. R., Horn, J. L. \& Nesselroade, J. E. (1969). Factor matching procedures. An improvement of the $\mathrm{s}$ index, with tables. Educational and Psychological Measurement, 29, 781-792.

Devine, P. G. \& Elliot, A. J. (1995). Are racial stereotypes really fading? The Princeton trilogy revisited. Personality and Social Psychology Bulletin, 21, 1139-1150.

Dovidio, J. F. \& Gaertner, S. L. (1998). On the nature of contemporary prejudice: The causes, consequences, and challenges of aversive racism. En J. L. Eberhardt \& S. T. Fiske (Eds.), Confronting racism: The problem and the responses (pp. 3-32). Thousand Oaks, CA: Sage.

Fiske, S. T., Cuddy, A. J. C., Glick, P. \& Xu, J. (2002). A model of (often mixed) stereotype content: Competence and warmth respectively follow from perceived status and competition. Journal of Personality and Social Psychology, 82, 878-902.

Foerster, R. \& Vergara, J. I. (2003). Etnia y nación en la lucha por el reconocimiento: los mapuches en la sociedad chilena. En H. Gundermann, R. Foerster \& J. I. Vergara (Eds.), Mapuches y aymaras: el debate en torno al reconocimiento y los derechos ciudadanos (pp. 105-177). Santiago, Chile: PREDES \& Ril.

Haslam, S. A., Turner, J. C., Oakes, P. J. \& McGarty, C. (1992). Context-dependent variation in social stereotyping. I: The effects of intergroup relations as mediated by social change and frame of reference. European Journal of Social Psychology, 22, 3-20.

Hewstone, M. (1994). Revision and change of stereotypic beliefs: In search of the elusive subtyping model. En W. Stroebe \& M. Hewstone (Eds.), European review of social psychology (Vol. 5, pp. 69-109). Chichester, Inglaterra: Wiley.

Instituto Nacional de Estadística. (2005). Estadísticas sociales de los pueblos indigenas en Chile - Censo 2002. Santiago, Chile: Autor.

LeVine, R. A. \& Campbell, D. T. (1972). Ethnocentrism: Theories of conflicts, ethnic attitudes, and group behavior. New York: Wiley.

Marimán, P., Canuiqueo, S., Millalén, J. \& Levil, R. (2006). i...Escucha, winka...! Cuatro ensayos de historia nacional mapuche y un epílogo sobre el futuro. Santiago, Chile: LOM.

McCabe, A. E. \& Brannon, L. A. (2004). An examination of racial subtypes versus subgroups. Current Research in Social Psychology, 9, 109-123.

Merino, M. E., Millamán, R., Quilaqueo, D. \& Pilleux, M. (2004). Perspectiva interpretativa del conflicto entre mapuches y no mapuches sobre la base del prejuicio y discriminación étnica. Persona y Sociedad, 18(1), 111-128.

Merino, M. E. \& Quilaqueo, D. (2003). Ethnic prejudice against the Mapuche in Chilean society as a reflection of the racist ideology of the Spanish conquistadors. American Indian Culture and Research Journal, 27(4), 105-116.

Naguil, V. (1999). Conflictos en el territorio mapuche: intereses, derechos y soluciones políticas en juego. Liwen, 5, 11-41.

Nelson, T. D. (2006). The psychology of prejudice. Boston, MA: Pearson Education.

Olzak, S. (1992). The dynamics of ethnic competition and conflict. Stanford, CA: Stanford University.

Park, B., Ryan, C. S. \& Judd, C. M. (1992). Role of meaningful subgroups in explaining differences in perceived variability for the in-groups and out-groups. Journal of Personality and Social Psychology, 63, 553-567.

Pilleux, M. (2002). La educación y el prejuicio étnico. Contexto Educativo, 24. Extraído el 2 Diciembre, 2007, de http:// contexto-educativo.com.ar/2002/4/nota-07.htm 
Quilaqueo, D. \& Merino, M. E. (2003). Estereotipos y prejuicio étnico hacia los mapuches en textos complementarios a la asignatura de Historia. Campo Abierto, 23, 119-135.

Richards, Z. \& Hewstone, M. (2001). Subtyping and subgrouping: Processes for the prevention and promotion of stereotype change. Personality and Social Psychology Review, 5, 52-73.

Rojas, M. \& Sepúlveda, S. (2002). Prejuicio étnico respecto de profesores mapuches de la ciudad de Teтuco. Tesis no publicada para optar al grado de Licenciado en Educación, Universidad Católica de Temuco, Chile.

Saavedra, A. (2002). Los mapuches en la sociedad chilena actual. Santiago, Chile: LOM.

Saiz, J. L. (1991). Current stereotypes of past and present South American Indians. Tesis no publicada para optar al grado de Master of Arts in Psychology, Wake Forest University, Winston-Salem, NC, Estados Unidos.

Saiz, J. L. (2002). Atribución de estereotipos: los indígenas mapuches que perciben los chilenos. En J. F. Morales, D. Páez, A. L. Kornblit \& D. Asún (Eds.), Psicología social (pp. 145-151). Buenos Aires: Prentice Hall-Pearson Educación.

Saiz, J. L., Merino, M. E. \& Quilaqueo, D. (en prensa). Metaestereotipos sobre los mapuches de Chile. Interdisciplinaria.
Saiz, J. L. \& Williams, J. E. (1991). Estereotipos del indígena mapuche: una verificación empírica de proposiciones y hallazgos previos. En Y. Kuramochi \& P. de la Peña (Eds.), Sobre culturas indigenas: lenguaje e identidad (pp. 141-148). Temuco, Chile: CONICYT-UCT-UFRO.

Schneider, D. J. (2005). The psychology of stereotyping. New York: The Guilford Press.

Sherif, M. (1966). Group conflict and co-operation: The Robber's cave experiment. London: Routledge \& Kegan Paul.

Tabachnick, B. G. \& Fidell, L. S. (1989). Using multivariate statistics. New York: Harper Collins.

ten Berge, F. M. F. (1977). Optimizing factorial invariance. Groningen, Holanda: VRB Drukkerijen.

von Baer, E. (2004). A diez años de la Ley Indígena: ¿Qué piensan los mapuches de las comunidades rurales? (Serie Informe Político $\mathrm{N}^{\mathrm{o}}$ 82). Santiago, Chile: Instituto Libertad y Desarrollo.

Yáñez, N. \& Aylwin, J. (Eds.) (2007). El gobierno de Lagos, los pueblos indigenas y el "nuevo trato". Santiago, Chile: LOM.

Fecha de recepción: Marzo de 2008.

Fecha de aceptación: Septiembre de 2008. 